\title{
Estructura y tipologías de la práctica de lo paranormal: un análisis matemático-formal de sus productos cognitivos
}

\author{
ANTONIO VELASCO CASTRO
}

Las prácticas de lo paranormal son un fenómeno universal para el control y el conocimiento de sí mismo, los semejantes y el entorno, en el que el mundo se asume como dependiente de la agencia contraintuitiva y no evidente a los sentidos. Aquí se parte de un análisis racional de las explicaciones que las personas dan a los fenómenos de su vida diaria y se presenta un modelo de los componentes, clases, tipos y formas de lo paranormal. Se analizan las principales tipologías en la materia y se postula un modelo según el cual el núcleo de la práctica paranormal está compuesto por organismo, eventos y conducta, así como sus relaciones funcionales, de lo cual surgen ciertas estructuras. Se encontraron cuatro clases fundamentales, 24 tipos y 12 formas de las prácticas de lo paranormal.

PALABRAS CLAVE: brujería, magia, paranormal, sobrenatural, superstición

Structure and Typologies of Paranormal Practices: A Mathematical Analysis of their Cognitive Products

The practices of the paranormal are a universal phenomenon, used for the control and knowledge of oneself, the peers and the environment. The world is assumed as dependent on counterintuitive agency and non-evident to the senses. In this work a rational analysis is made on the explanations that people give to the phenomena of their daily life, and a model is presented on the components, classes, types and forms of the paranormal. The main typologies in the subject are analyzed, followed by a model according to which the core of the practices of the paranormal is composed of organism, events and behavior, as well as their functional relationships, resulting in the conformation of structures. Four fundamental classes were found, with 24 types and 12 forms of paranormal practices. 


\section{Introducción}

a existencia de lo paranormal se acepta quizá en todas las culturas y países, aunque su función y significado difiere de una cultura a otra, así como de una comunidad a otra, e incluye una variedad de fenómenos en contradicción con las pautas aparentes de la percepción, aun cuando se presenta bajo diversas etiquetas y manifestaciones concretas en todas las épocas (George y Sreedhar, 2006; Murray, 2006; Näyhä, 2002).

Al tratarse de un fenómeno generalizado, debe existir una estructura básica que origine los tipos del fenómeno, mas no existe un modelo de aceptación general que proponga una tipología que no sea simplemente intuitiva ni puramente casuística sobre cuáles son los elementos básicos de la práctica en cuestión, o sobre qué relaciones se presentan entre ellos y a qué tipos dan lugar.

Por esta razón, se efectúa un análisis matemático-formal de ciertas estructuras cognitivas producto de la práctica, de modo que en un sentido amplio puede decirse que el presente es un enfoque estructuralista, que al ser confrontado con los hechos entra en el ámbito de la etnociencia. Pasar de la pura formalización al análisis de la realidad antropológica implica que las estructuras formales deben ser adaptadas a los hechos culturales y no al revés: lo importante es que los modelos se creen a partir de hechos reales.

Las interrogantes que han guiado la investigación han sido las siguientes: 1) ¿cuál es la estructura mínima posible que permite representar las prácticas de lo paranormal?, y 2) ¿qué características debe tener una tipología de tales prácticas? A partir de ello, se propone un modelo matemático sencillo, integrado por elementos básicos, parámetros, relaciones funcionales y tipologías. ${ }^{1}$

$1 \quad$ Por razones de espacio, el análisis que se presenta es totalmente teórico, aunque basado en investigación y trabajo de campo sobre las creencias en lo paranormal. 
En este artículo no se toma partido respecto de las importantes polémicas que ocupan el campo antropológico actual, sea en la ciencia cognitiva de la religión o en la antropología posthumanista y no racionalista, pues el análisis formal no entra per se en conflicto con ningún enfoque epistemológico.

\section{La práctica como formato general}

\section{IDEA DE LA PRÁCTICA}

El concepto de práctica proporciona el formato adecuado para la comprensión de actividades que presentan un parecido de familia, como la magia, la religión y la superstición, en cuanto que todas apelan a la agencia contraintuitiva y a fenómenos no evidentes a los sentidos. Una práctica se define como cualquier forma coherente y compleja de actividad humana cooperativa, socialmente establecida, mediante la que se realizan los bienes inherentes a ella, mientras se intentan lograr los modelos de excelencia que le son apropiados (MacIntyre, 1981).

En una práctica se pueden identificar elementos que definen su estructura, a saber: 1) sujetos, que a su vez generan ciertos elementos: un sistema de creencias, los fines que se desea alcanzar con la práctica, las razones para ejecutarla —individuales y sociales-; 2) modos de ejecución, conductas o procedimientos requeridos; 3) entorno o contextos pertinentes; 4) propósito, y 5) condiciones de ejecución - tiempo, lugar, instrumentos y modo de ejecución-.

\section{LAS PRÁCTICAS DE LO PARANORMAL²}

El ámbito de lo paranormal incluye actividades que presentan elementos en común y son prácticas por derecho propio - como la religión y la magia-, de modo que su denominación con etiquetas como las de superstición, brujería o hechicería resulta sesgada, y la distinción entre las prácticas así etiquetadas puede ser arbitraria. Tales prácticas configuran una familia cuyo mínimo denominador común es la creencia en la agencia contraintuitiva y no evidente a los sentidos; más allá de ello, desde luego existen importantes diferencias. ${ }^{3}$

\section{Modelos y tipologías acerca de lo paranormal}

\section{TIPOLOGÍAS DE LA MAGIA}

La tipología estándar de la magia entre finales de la Edad Media y comienzos del Renacimiento se encuentra en el Malleus maleficarum - primera parte, quaestio 16-, de 1486; sus autores, Heinrich Kramer y Jacobus Sprenger (1996), dicen que existen 14 tipos de magia, que surgen de tres clases de adivinación.

Una tipología renacentista es la de Giordano Bruno (2007), quien a finales del siglo XVI, luego de exponer las acepciones populares del término, expresa que, en correspondencia con los mundos arquetípico, físico y racional, hay tres tipos de magia: divina, natural o física, y matemática; esta última, por las disciplinas matemáticas de la época —óptica, música, geometría- y por ser intermediaria entre las operaciones divinas y las naturales. Las dos primeras son necesariamente buenas y la tercera puede no serlo, según quien la emplee.

2

Para hacer referencia al fenómeno en general, se hablará de práctica; para sus manifestaciones concretas, se hablará de prácticas.

$3 \quad$ Si se asume una postura de comprensión de estas prácticas desde dentro del canon cultural occidental contemporáneo - etic-, se considerará que las prácticas carecen de fundamento; pero si se asume una postura desde fuera de dicho canon, o dentro de uno empático con lo paranormal -emic-, se podrá considerar que las prácticas tienen un fundamento adecuado (Frater, 2005; Greenwood, 2000) 


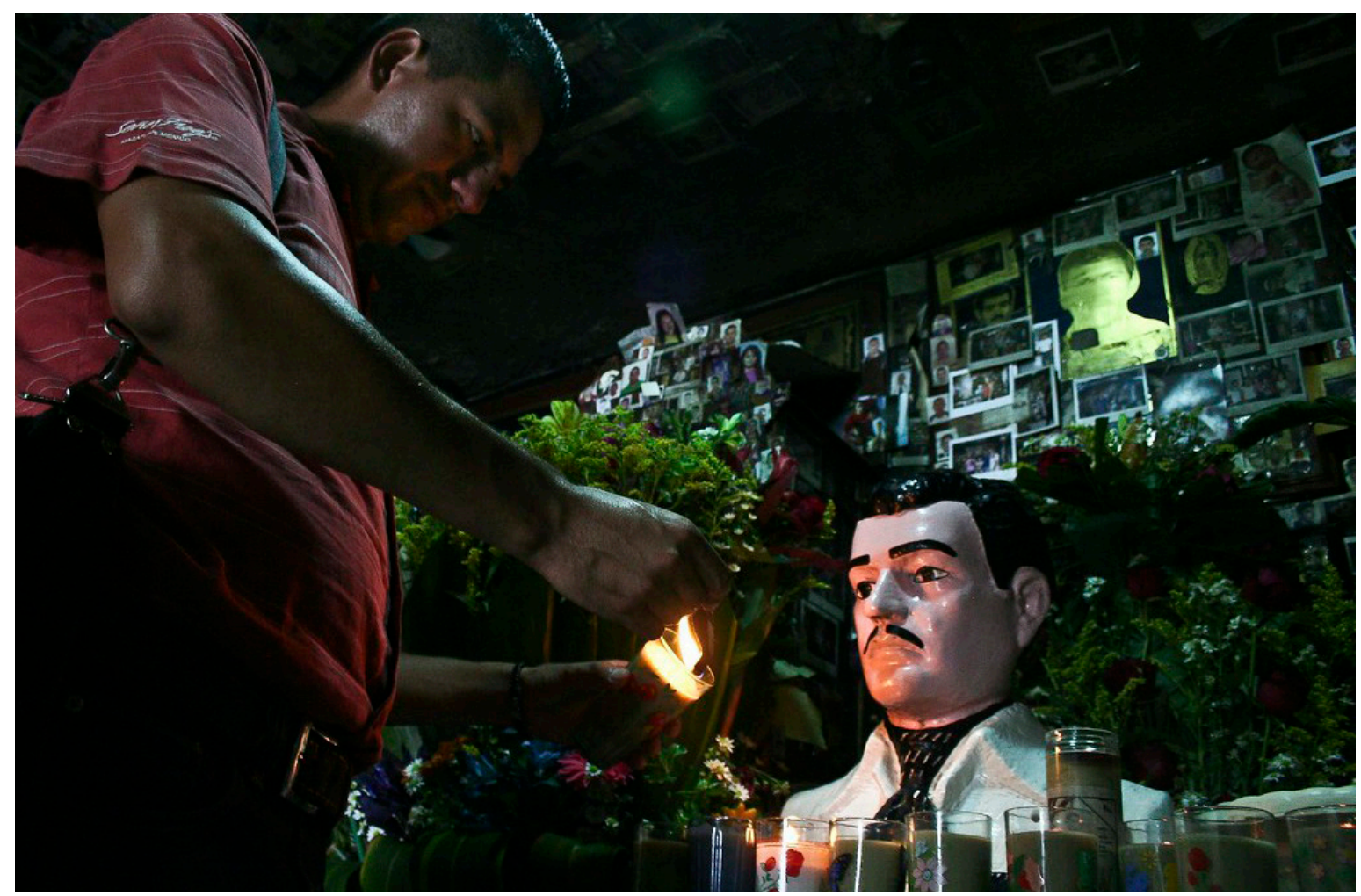

VIDEXISTENZ/FLICKR • Devoto de Jesús Malverde.

En la época contemporánea, James Leuba (1912) propuso una clasificación de la magia según tres principios: 1) magia por repetición - lo que sucede una vez posiblemente se repetirá-; 2) magia simpática — principio de transmisión del efecto- - que se compone de asociación por similitud entre objetos, asociación por contiguiidad y relación causal telepática entre objetos, y 3) magia de poder — principio de eficiencia de la voluntad-. ${ }^{4}$

Hoy en día, Frater (2005) expone cinco modelos — también les llama paradigmas — de la magia. El primero es el modelo espiritual, el más antiguo, que se puede encontrar en todas partes, tanto en el chamanismo como en la religión. Su premisa básica es la existencia de un mundo alterno habitado por entidades más o menos autónomas — espíritus, ángeles, demonios, dioses, entre otros- El segundo modelo es el de la energía, surgido con el mesmerismo. El tercero es el modelo psicológico, en el que el subconsciente y el inconsciente harán su labor si se programan de manera adecuada. El cuarto modelo es el de la información, que no está sujeta a las limitaciones de la materia ni de la energía y permite controlar el flujo de la energía. El quinto es un metamodelo para el manejo de los anteriores, cuya premisa sería utilizar siempre el modelo más adecuado al propósito.

Finalmente, ante la diversidad semántica, la variedad de tipologías y las proposiciones de eliminar el

4

Leuba (1912) no utiliza las denominaciones de magia por repetición y magia de poder, aquí se las propone como etiquetas de clasificación. 
concepto del discurso académico, Bernd-Christian Otto y Michael Stausberg (2014) han optado por renunciar a una definición de lo que denominan "magia" y proponen una tipología puramente casuística, lo cual, en realidad, no pone orden en el tema, sino que perpetúa la anarquía tipológica.

\section{MODELOS Y TIPOLOGÍAS DE LA SUPERSTICIÓN}

Existen tres tendencias en los modelos explicativos de la superstición (S). La primera es la de los modelos causalistas y correlacionalistas, que se centran en la relación entre conducta y eventos ambientales sobre la base de la atribución errónea de causalidad, la correlación ilusoria y la ilusión de control (Pronin et al., 2006). Según esta tendencia, ciertos eventos se pueden predecir dadas ciertas conductas, o bien explicarse si ya ocurrieron - "retrodecir"- y predecir, dados ciertos eventos. Aquí se encuentran los modelos evolucionistas de Kevin Foster y Hanna Kokko (2009), y Jan Beck y Wolfgang Forstmeier (2007), así como los modelos conductistas de Burrhus Skinner (1948) y Helena Matute (Matute y Vadillo, 2012; Vadillo, Matute y Blanco, 2013).

La segunda es la del procesamiento de información según el sistema de creencias, el procesamiento dual y la confusión ontológica, que corresponden a los modelos de pensamiento mágico de Thomas Markle (2010), el cognitivista de Marjaana Lindeman y Kia Aarnio (2007) y la teoría sociológica cognitiva de la semicreencia de Colin Campbell (1996).

La tercera corriente es mixta e incluye el modelo de necesidades, el modelo de Dundes (1961; D’Agati, 2014) y los modelos psicológicos del trastorno obsesivo compulsivo (TOC; Brugger y Viaud-Delmont, 2010; Simonds, Demetre y Read, 2009).

Por otro lado, la tipología de la superstición de Peter Jarvis (1980) incluye: 1) presagios o signos para la predicción de vida, muerte y suerte buena o mala; 2) tabúes o sanciones negativas, prohibiciones, y 3) hechizos, fórmulas para generar vida o muerte, evitar mala suerte o crear buena suerte. Finalmente, la clasificación de László Sándor (2007: 104-107) propone: 1) observación — de signos y temporal—; 2) adivinación, y 3) magia.

Como se puede apreciar, se echa en falta tanto un criterio que unifique las tipologías, como una estructura o núcleo mínimo que fundamente y brinde coherencia a las clasificaciones, lo que se propondrá en seguida.

\section{El núcleo}

La práctica de lo paranormal $(\mathrm{P})$, como hechicería, religión, chamanismo, curanderismo, brujería, magia, superstición y similares, se fundamenta en una relación simbólica entre un organismo y su entorno, en la que aquél cree que puede controlar, predecir, conocer o vivenciar a éste y a sí mismo mediante su conducta o su interpretación.

El conjunto de agentes se representará con la letra $\mathrm{O}$, lo que incluye a $\mathrm{O}$-organismo animado, capaz de intencionalidad-, O' — sus semejantes-, e I —agentes invisibles-. El conjunto de sus conductas - acción u omisión - se representará con la letra $\mathrm{C}$, y el conjunto de eventos, entes y cosas de interés para $\mathrm{O}$, del entorno — natural y sobrenatural-, se representará con la letra $\mathrm{E}$, de manera que: $\mathrm{P}=\langle\mathrm{O}, \mathrm{C}, \mathrm{E}\rangle$.

Las relaciones funcionales entre los elementos de P ocurren así: el entorno E está compuesto por una serie de fenómenos, eventos, cosas, sucesos $\left(E_{m} \ldots E_{n}\right)$, incluyendo a cualesquiera otros agentes y sus conductas, ${ }^{5}$ y el conjunto $\mathrm{C}$ se compone de

5

Cuando la conducta del caso sea ejecutada por otro sujeto $\left(\mathrm{O}^{\prime}\right)$, ésta también puede ser considerada como perteneciente a una $\mathrm{C}$. 
series de conductas de $\mathrm{O}\left(\mathrm{C}_{\mathrm{m}} \ldots \mathrm{C}_{\mathrm{n}}\right) \cdot{ }^{6}$ El resultado de toda operación $\mathrm{P}$ se manifiesta en $\mathrm{E}$ o en el propio $\mathrm{O}$, aunque, para simplificar, en adelante se hablará de resultados en E.

Algunos componentes de $\mathrm{E}$ - son generados por uno o más de los elementos de C, ya sea por uno solo, como en el caso de un gesto simple o una conducta dada, $\mathrm{C}_{\mathrm{n}}$, o bien por una serie de ellos, $\mathrm{C}_{\mathrm{m}} \ldots \mathrm{C}_{\mathrm{n}}$, como la ejecución de un ritual o la preparación de un brebaje mágico. Las relaciones funcionales y su papel en la producción de P se explicarán luego de examinar sus orígenes.

\section{Origen de las $\mathrm{P}$}

\section{ORIGEN DE LAS RELACIONES DE C EN E}

Existe una relación entre $\mathrm{C}$ y E, donde uno o más elementos de $\mathrm{C}$ se considera causa de uno o más elementos de E. Así, una serie de eventos ambientales - de la serie $\mathrm{E}$ - puede estar asociada aleatoriamente a cierta conducta — de la serie C - de un $\mathrm{O}$; por ejemplo, el hecho de que usualmente, o al menos en ciertas ocasiones, llueva cuando una persona pone al sol ropa lavada o cuando un aborigen baila cierta danza; el que una paloma comúnmente reciba alimento cuando ejecuta cierta conducta en un laboratorio, etcétera.

Así, el psicólogo Skinner (1948), en sus experimentos de mediados del siglo xx, observó cómo ciertas conductas estereotipadas y causalmente ineficaces podían aparecer en los organismos si habían sido seguidas por reforzamientos adecuados, y las denominó “superstición”.

Tales situaciones pueden llegar a ser culturalmente típicas, y el organismo aprende por sí mismo esa relación entre E y C: su aparato mental archiva los E y los relaciona con situaciones nuevas pero similares — asimilables a la serie E-, que de manera progresiva aparecen en el transcurso de su existencia; o bien, se le enseña al sujeto, por aprendizaje cultural en la manada, la familia, la escuela, la calle, etc., que tales situaciones guardan alguna relación entre sí.

Lo relevante es que en algún momento el organismo termina asociando dicha serie de eventos con alguna o algunas $\mathrm{C}_{\mathrm{n}}$ de $\mathrm{C}$, y llega a considerar que, si ejecuta una conducta como ésa o alguna asimilable, el evento correspondiente ocurrirá. Se trata de lo que se denomina "condicionamiento operante": se ejecuta una conducta, ocurre un evento ambiental $n$ veces, y el organismo aprende a esperar que esa relación se mantenga y actuar en consecuencia.

Esto sucede para un fenómeno que constituye un primer grupo de las $\mathrm{P}$, o $\mathrm{P}$ clase $\alpha\left(\mathrm{P}_{\alpha}\right)$ : en ésta se presenta una relación entre dos elementos, el organismo y su entorno, tal que aquél depende de éste para la obtención de sus fines, mediante la ejecución de conductas ritualistas, basadas en interpretaciones estereotipadas acerca del funcionamiento de sí mismo, del entorno y de su relación con éste.

Se presentaría así una atribución errónea de causalidad, puesto que el fenómeno considerado como causante carecería de eficacia causal para producir el evento con el que se le relaciona (Pronin et al., 2006). De esta manera, se crea un ciclo psicológicamente autosatisfactorio - profecía autocumplida-, debido a que cada vez que sucede $\mathrm{E}_{\mathrm{n}}$ se recuerda $C_{n}$, o cada vez que se ejecuta $C_{n}$ se espera $\mathrm{E}_{\mathrm{n}}$, mientras se desecha cognitivamente las ocasiones en las que la relación no se cumple.

Así pues, según su aprendizaje, el organismo tenderá a recordar la relación más saliente en su memoria, la que asocia la serie E a la serie C, y desechará como irrelevantes o no tomará en cuenta los casos en los que tal relación no se cumple. He allí el origen de $\mathrm{P}_{\alpha}$.

La cuestión de qué se considera una conducta concreta - acotación de la conducta- depende de la intencionalidad de $\mathrm{O}$ y la intencionalidad social - como en el caso de los ritos-, algo que no será analizado aquí. 


\section{ORIGEN DE LAS RELACIONES DE E EN E}

En este caso, la relación no es entre conducta y eventos, sino entre eventos, de modo que la ocurrencia o ausencia de alguno se considera como señal de la ausencia u ocurrencia de otro con el cual se le relaciona; por ello, al no existir una relación causal, lo que ocurre es una correlación ilusoria entre eventos.

A este segundo grupo de $\mathrm{P}$ se le denominará $\mathrm{P}$ clase $\beta\left(\mathrm{P}_{\beta}\right)$ : en él se encuentran fenómenos como la interpretación de augurios favorables o desfavorables y las mancias, como la lectura de las cartas, las manos o los espejos (Melchior-Bonnet, 2001; Stevens, 2004). En este caso, según el aprendizaje personal, social o ambos, el organismo tenderá a recordar ciertas relaciones entre eventos, aquellas que correlacionan una serie E con otra, lo que le permite formular predicciones y retrodicciones, mientras desecha como irrelevantes los casos en los que tal relación no se cumple o parece no cumplirse. Éste es el origen de $\mathrm{P}_{\beta}$.

\section{ORIGEN DE LAS RELACIONES DE C EN E}

Este tercer grupo de $\mathrm{P}$ se denominará $\mathrm{P}$ clase $\chi\left(\mathrm{P}_{\chi}\right)$ y se refiere a los procesos de pensamiento y conducta involucrados en el TOC. La asociación entre el evento en cuestión y su extensión a una serie de eventos similares $\left(\mathrm{E}_{\mathrm{m}} \ldots \mathrm{E}_{\mathrm{n}}\right)$ da lugar a un incremento de la ansiedad que interfiere con la actividad normal del sujeto y se manifiesta como obsesión acerca de algo y como la creencia de que ello puede producirse o evitarse mediante la omisión o ejecución reiterada de una conducta, lo que brindará alivio al disminuir la ansiedad.

Lo que caracteriza al TOC como una $\mathrm{P}$ es: a) presencia de un pensamiento obsesivo - intrusivo y persistente-, según el cual cierto evento deseable o indeseable depende de C; b) ejecución compulsiva de una conducta, esto es, indefinidamente repetitiva, en un ciclo que al sujeto le cuesta mucho romper, y c) la atribución causal entre la conducta compulsiva y el evento es considerada por el individuo como ilógica pero causalmente eficaz (Spears, 2013).

\section{ORIGEN DE LAS RELACIONES ENTRE LA EXPERIENCIA Y LO INEFABLE}

Un cuarto grupo de $\mathrm{P}$ se denominará $\mathrm{P}$ clase $\delta\left(\mathrm{P}_{\delta}\right)$ y correponde a los procesos en los cuales $\mathrm{O}$ experimenta o presencia lo que en ese momento considera paranormal. La asociación entre lo que resulta inexplicable $(\Omega)$ y la experiencia del sujeto (Exp) se manifiesta como un medio para $\mathrm{P}$, o bien como el atestiguamiento de $\mathrm{P}$, se encuentre o no $\mathrm{O}$ en capacidad de explicar en forma racional el fenómeno experimentado o atestiguado.

En esta oportunidad, se trata de cuestiones tales como las siguientes: a) por una parte, los casos en los que un sujeto aparentemente sirve como medio para la manifestación o la actividad de algo, como por ejemplo en la posesión, mediumnidad, escritura automática o trance oracular (Oberter, 2012); y b) los casos en los que $\mathrm{O}$ percibe la ocurrencia de algo que, al menos en principio, califica como una experiencia $\mathrm{P}$, lo sea o no, como ocurre en los contextos en los cuales una persona es testigo de lo que considera que es $\mathrm{P}$, de modo que esta clase de $\mathrm{P}$ surge de la vivencia personal en la que $\mathrm{O}$ le otorga algún sentido a lo vivido, frente a la inexplicabilidad - real o aparente- de P.

\section{Relaciones entre los elementos de $\mathrm{P}$}

\section{FUNCIONES}

Una primera relación funcional entre C y E es de tipo causal: $\mathrm{O}$ asume que la ejecución o cesación de una conducta dada — por su parte o por parte de 
O'- genera uno o más de los elementos de E, aunque la relación en realidad sea causalmente ineficaz - atribución errónea de causalidad-. En este caso, se trata de una función $\kappa$, según la cual E, $\mathrm{O}=\kappa(\mathrm{C}){ }^{7}$

Una segunda relación funcional en $\mathrm{P}$ se presenta dentro del propio conjunto E, y es de tipo correlacional: $\mathrm{O}$ asume que la ocurrencia o cesación de un evento dado - o una serie de ellos- se encuentra relacionada con uno o más elementos de E. Por ejemplo, la interpretación de los augurios - hermenéutica paranormal de los eventos- o las mancias - hermenéutica paranormal de los objetos- En este caso, se da una función $\rho$, según la cual $\mathrm{E}=\rho(\mathrm{E})$.

Una tercera relación funcional entre $\mathrm{C}$ y E es también de tipo causal, pero presenta dos características especiales: $\mathrm{O}$ asume que la ejecución o cesación de una conducta suya genera o anula uno o más de los elementos de E, pero además de que la relación sea causalmente ineficaz, no ocurre realmente una atribución errónea de causalidad, puesto que el sujeto está consciente de la ineficacia causal de su conducta, mas le resulta en extremo difícil dejar de ejecutarla, debido a la creencia en que su conducta es realmente eficaz de modo inexplicable e incomprensible. En este caso, es la función ఐ, según la cual $\mathrm{E}, \mathrm{O}=\varpi(\mathrm{C})$.

Nótese que en las relaciones funcionales de tipo causal, $\mathrm{O}$ es un agente generador o finalizador de eventos o cuestiones en el mundo - lo que lo incluye a él y a O'- que actúa sobre el entorno o sobre sí mismo, en tanto que en las relaciones de tipo correlacional, $\mathrm{O}$ es un hermeneuta paranormal, esto es, un intérprete de ciertos signos que se presentan en el entorno - que incluyen cualesquiera cosas materiales, eventos y agentes-.

La cuarta relación funcional que se presenta es una en la que la vivencia de $\mathrm{O}$ es un resultado por transducción - conversión de una cosa en otrade lo inexplicable o inefable $(\Omega)$ en explicable, aunque ello sea de modo parcial o temporal. Ante una experiencia $\mathrm{P}$, las personas procuran encontrar explicaciones que se ajusten a sus esquemas de comprensión, sean correctas o no. En este caso, se trata de la función $\psi$, según la cual $\operatorname{Exp}=\psi(\Omega)$.

\section{PRIMERA CLASE DE RELACIONES: RELACIONES DE CAUSALIDAD DE C HACIA E (P CLASE $\alpha)^{8}$}

Según $\mathrm{O}$, su $\mathrm{C}_{\mathrm{n}}$ provoca un $\mathrm{E}_{\mathrm{n}}$ favorable $\left(\mathrm{E}^{+}\right)$, por ejemplo, creer el bateador que golpear tres veces la suela del zapato izquierdo con la punta del bate, inmediatamente antes de batear, le permitirá conectar un jonrón. Pero también hay casos en los que, según $\mathrm{O}$, una conducta diferente, como $\mathrm{C}_{\mathrm{m}}$, hace finalizar un $\mathrm{E}_{\mathrm{n}}$ desfavorable $\left(\mathrm{E}^{-}\right)$, por ejemplo, colocar un vaso de agua tras una puerta a fin de que una visita indeseable se vaya.

Siendo así, hay dos clases de C en P de carácter causal: una conducta generativa, que la persona cree que provoca o genera eventos (E) y que se simbolizará como $\mathrm{Cg}$, y otra conducta anuladora, que el sujeto cree que evita o finaliza eventos $(\overline{\mathrm{E}})$ que se simbolizará como Ca. Consecuentemente, se presentan también la ausencia de $\mathrm{Cg}(\overline{\mathrm{C}} \mathrm{g})$ y de $\mathrm{Ca}(\overline{\mathrm{C}} \mathrm{a})$, es decir, las conductas de omisión o cesación de las dos anteriores, respectivamente. Así, existen cuatro conjuntos de $\mathrm{C}$ ejecutada: $\mathrm{Cg}=\left(\mathrm{Cg}_{1} \ldots \mathrm{Cg}_{\mathrm{n}}\right)$ y $\mathrm{Ca}=\left(\mathrm{Ca}_{1} \ldots \mathrm{Ca}_{\mathrm{n}}\right)$, así como los conjuntos de conductas omisivas o no ejecutadas: $\overline{\mathrm{C}} \mathrm{g}=\left(\overline{\mathrm{C}} \mathrm{g}_{1} \ldots \overline{\mathrm{C}} \mathrm{g}_{\mathrm{n}}\right)$ y $\overline{\mathrm{C}} \mathrm{a}=\left(\overline{\mathrm{C}} \mathrm{a}_{1} \ldots \overline{\mathrm{C}}_{\mathrm{n}}\right)$.

En ambos casos - generación y anulación-, $\mathrm{O}$ cree que existe una relación causalmente eficaz entre C y E. La dirección causal va desde C hasta $\mathrm{E}$, y la función que caracteriza la relación entre C y

\footnotetext{
$7 \quad$ La invocación de entidades sobrenaturales es un caso de este tipo, porque, aunque el ente causal no sería O, la actuación de aquella entidad es provocada por 0 .

8 Por razones de espacio, no se incluirán ejemplos en los cuadros.
} 
CUADRO 1. TIPOS DE LA CLASE S SEGÚN LA RELACIÓN CAUSAL K

\section{Eventos en el mundo}

\section{Generar eventos}

\section{Anular eventos}

\begin{tabular}{lccccc}
\hline Conductas de $\mathrm{O}$ & & favorables $\mathrm{E}^{+}$ & desfavorables $\mathrm{E}^{-}$ & favorables $\overline{\mathrm{E}}^{+}$ & desfavorables $\overline{\mathrm{E}}^{-}$ \\
\hline $\begin{array}{l}\text { Conductas } \\
\text { generadoras }\end{array}$ & $\mathrm{Cg}$ & $\mathrm{P}_{1}$ & $\mathrm{P}_{2}$ & $\mathrm{P}_{\mathrm{e}}$ & $\mathrm{P}_{\mathrm{f}}$ \\
\hline \multirow{2}{*}{$\begin{array}{l}\text { Conductas } \\
\text { anuladoras }\end{array}$} & $\overline{\mathrm{C}}$ & $\mathrm{P}_{\mathrm{a}}$ & $\mathrm{P}_{\mathrm{b}}$ & $\mathrm{P}_{3}$ & $\mathrm{P}_{4}$ \\
\cline { 2 - 6 } & $\overline{\mathrm{C}} \mathrm{a}$ & $\mathrm{P}_{\mathrm{c}}$ & $\mathrm{P}_{\mathrm{d}}$ & $\mathrm{P}_{5}$ & $\mathrm{P}_{6}$ \\
\hline
\end{tabular}

Fuente: Elaboración propia.

\section{CUADRO 2. DESCRIPCIÓN DE LOS TIPOS DE P}

\begin{tabular}{cll} 
Tipos & $\begin{array}{c}\text { Relación funcional } \\
\mathbf{E}=\kappa(\mathrm{C})\end{array}$ & \multicolumn{1}{c}{ Significado } \\
\hline$P_{1}$ & $\mathrm{E}^{+}=\kappa(\mathrm{Cg})$ & Una conducta generadora origina un evento positivo. \\
\hline$P_{2}$ & $\mathrm{E}^{-}=\kappa(\mathrm{Cg})$ & Una conducta generadora origina un evento negativo. \\
\hline$P_{3}$ & $\bar{E}^{+}=\kappa(\overline{\mathrm{C} g})$ & $\begin{array}{l}\text { La finalización -omisión o cesación }- \text { de una conducta generadora causa } \\
\text { el final de un evento positivo. }\end{array}$ \\
\hline$P_{4}$ & $\bar{E}^{-}=\kappa(\bar{C} g)$ & $\begin{array}{l}\text { La finalización -omisión o cesación- de una conducta generadora causa } \\
\text { el final de un evento negativo. }\end{array}$ \\
\hline$P_{5}$ & $\bar{E}^{+}=\kappa(C a)$ & Una conducta anuladora finaliza o frustra un evento positivo. \\
\hline$P_{6}$ & $\bar{E}^{-}=\kappa(C a)$ & Una conducta anuladora finaliza o evita que ocurra un evento negativo. \\
\hline$P_{7}$ & $E^{+}=\kappa(\bar{C} a)$ & $\begin{array}{l}\text { La eliminación de una conducta anuladora causa o permite que continúe } \\
\text { un evento positivo. }\end{array}$ \\
\hline$P_{8}$ & $E^{-}=\kappa(\bar{C} a)$ & $\begin{array}{l}\text { La eliminación de una conducta anuladora causa o permite que continúe } \\
\text { un evento negativo. }\end{array}$ \\
\hline
\end{tabular}

Fuente: Elaboración propia.

E, como ya se dijo, es una relación causal ilusoria. De esta manera hay 16 clases de relaciones causales entre $\mathrm{C}$ y E en P; de ellas, ocho no son posibles — subíndices literales, casillas sombreadas-, y ocho son posibles — no se considera el factor tiempo- (véanse los cuadros 1 y 2).

\section{SEGUNDA CLASE DE RELACIONES: CORRELACIÓN DESDE E HACIA E (P CLASE $\beta$ )}

Los estados de cosas o eventos singulares $\left(\mathrm{E}_{\mathrm{n}} \ldots \mathrm{E}_{\mathrm{m}}\right)$ pertenecientes a la serie que compone el conjunto $\mathrm{E}$ pueden ser considerados favorables o desfavorables por $\mathrm{O}$, y los eventos referidos pueden ser ubicados temporalmente por $\mathrm{O}$ en el pasado o en el futuro, esto es, $\mathrm{P}$ puede referirse a eventos que ya sucedieron o que están por ocurrir.

En este caso, O recurre a un evento-signo en el presente, el pasado o el futuro - evento señalizador-, o bien a una configuración o forma de un objeto de interpretación - instrumento-, a los que se relaciona con un evento $\mathrm{E}_{\mathrm{m}}$ considerado favorable o desfavorable, ubicado en el presente, el pasado o el futuro. Como las relaciones de tipo $\kappa$ no son posibles aquí, se trata de correlaciones y no de relaciones 
CUADRO 3. TIPOS DE LA CLASE P ${ }_{\beta}$ SEGÚN LA RELACIÓN $\rho$

\section{Eventos}

\begin{tabular}{lccc}
\hline $\begin{array}{l}\text { Signos o eventos } \\
\text { señalizadores }\left(E^{s}\right)\end{array}$ & Pasados $(\mathrm{ps})$ & Presentes $(\mathrm{pt})$ & Futuros (ft) \\
\hline Favorables $\left(\mathrm{E}^{+}\right)$ & $\mathrm{P}_{9}$ & $\mathrm{P}_{10}$ & $\mathrm{P}_{11}$ \\
\hline Desfavorables $\left(\mathrm{E}^{-}\right)$ & $\mathrm{P}_{12}$ & $\mathrm{P}_{13}$ & $\mathrm{P}_{14}$ \\
\hline
\end{tabular}

Fuente: Elaboración propia.

\begin{tabular}{ccc} 
CUADRO 4. DESCRIPCIÓN DE LOS TIPOS DE $P_{\beta}$ & Significado \\
Tipo & $\begin{array}{c}\text { Relación funcional } \\
E_{n}=\rho\left(E^{s}\right)\end{array}$ & \\
\hline$P_{9}$ & $E_{p s}^{+}=\rho\left(E^{s}\right)$ & Un evento es señal de otro pasado, positivo o favorable. \\
\hline$P_{10}$ & $E_{p r}^{+}=\rho\left(E^{s}\right)$ & Un evento es señal de otro presente, positivo o favorable. \\
\hline$P_{11}$ & $E_{f t}^{+}=\rho\left(E^{s}\right)$ & Un evento es señal de otro futuro, positivo o favorable. \\
\hline$P_{12}$ & $E_{p s}^{-}=\rho\left(E^{s}\right)$ & Un evento es señal de otro pasado, negativo o desfavorable. \\
\hline$P_{13}$ & $E_{p r}^{-}=\rho\left(E^{s}\right)$ & Un evento es señal de otro presente, negativo o desfavorable. \\
\hline$P_{14}$ & $E_{f t}^{-}=\rho\left(E^{s}\right)$ & Un evento es señal de otro futuro, negativo o desfavorable.
\end{tabular}

Fuente: Elaboración propia.

CUADRO 5. TIPOS DE P ${ }_{\chi}$ SEGÚN LA RELACIÓN CAUSAL ๘ ENTRE C Y E: [E = ๘(C)]

\section{Eventos en el mundo}

\begin{tabular}{|c|c|c|c|c|c|}
\hline & & \multicolumn{2}{|c|}{ Generar eventos } & \multicolumn{2}{|c|}{ Anular eventos } \\
\hline \multicolumn{2}{|c|}{ Conductas de $\mathrm{O}$} & favorables $\mathrm{E}^{+}$ & desfavorables $\mathrm{E}^{-}$ & favorables $\overline{\mathrm{E}}^{+}$ & desfavorables $\overline{\mathrm{E}}$ \\
\hline \multirow{2}{*}{$\begin{array}{l}\text { Conductas } \\
\text { generadoras }\end{array}$} & $\mathrm{Cg}$ & $P_{15}$ & $P_{16}$ & $P_{m}$ & $P_{n}$ \\
\hline & $\overline{\mathrm{Cg}}$ & $P_{i}$ & $P_{j}$ & $P_{17}$ & $P_{18}$ \\
\hline \multirow{2}{*}{$\begin{array}{l}\text { Conductas } \\
\text { anuladoras }\end{array}$} & $\mathrm{Ca}$ & $P_{k}$ & $P_{1}$ & $P_{19}$ & $P_{20}$ \\
\hline & $\overline{\mathrm{C}} \mathrm{a}$ & $P_{21}$ & $P_{22}$ & $P_{0}$ & $P_{p}$ \\
\hline
\end{tabular}

Fuente: Elaboración propia.

causales, debido a que ambos eventos se encuentran ineludiblemente relacionados, aunque no sean generados uno por el otro: se trata de la correlación ilusoria entre eventos.

Se operan aquí dos clases de desplazamiento temporal: hacia el futuro (ft) — predicción- y hacia el pasado (ps)—retrodicción—, además de las relaciones que se asumen entre eventos simultáneos en el momento presente $(\mathrm{pt})$ —actualización—. Se trata de los siguientes casos: 1) signos del pasado; 2) signos del presente, y 3) augurios o presagios, en los cuales, con intención o carácter predictivo — profecías y similares—, o simultáneo o retrodictivo — visión del pasado-, se establecen o se asumen correlaciones entre eventos actuales y eventos presentes, futuros o pasados — signos del destino-. En el cuadro 3 se muestran las relaciones posibles, dados estos elementos, y en el cuadro 4, los tipos resultantes. 


\section{CUADRO 6. DESCRIPCIÓN DE LOS TIPOS DE $\mathrm{P}_{\chi}$}

\begin{tabular}{|c|c|c|}
\hline Tipos & $\begin{array}{l}\text { Relación funcional } \\
\qquad E=\varpi(C)\end{array}$ & Significado \\
\hline$P_{15}$ & $\mathrm{E}^{+}=\varpi(\mathrm{Cg})$ & Una conducta generadora origina un evento positivo. \\
\hline$P_{16}$ & $E^{-}=\varpi(C g)$ & Una conducta generadora origina un evento negativo. \\
\hline$P_{17}$ & $\overline{\mathrm{E}}^{+}=\varpi(\overline{\mathrm{C}} \mathrm{g})$ & $\begin{array}{l}\text { La finalización -omisión o cesación- de una conducta generadora causa } \\
\text { el final de un evento positivo. }\end{array}$ \\
\hline$P_{18}$ & $\overline{\mathrm{E}}^{-}=\varpi(\overline{\mathrm{C}} \mathrm{g})$ & $\begin{array}{l}\text { La finalización -omisión o cesación- de una conducta generadora causa } \\
\text { el final de un evento negativo. }\end{array}$ \\
\hline$P_{19}$ & $\overline{\mathrm{E}^{+}}=\varpi(\mathrm{Ca})$ & Una conducta anuladora finaliza o frustra un evento positivo. \\
\hline$P_{20}$ & $\overline{\mathrm{E}}^{-}=\varpi(\mathrm{Ca})$ & Una conducta anuladora finaliza o evita que ocurra un evento negativo. \\
\hline$P_{21}$ & $\mathrm{E}^{+}=\varpi(\overline{\mathrm{C}} \mathrm{a})$ & $\begin{array}{l}\text { La eliminación de una conducta anuladora causa o permite que continúe } \\
\text { un evento positivo. }\end{array}$ \\
\hline$P_{22}$ & $E^{-}=\varpi(\bar{C} a)$ & $\begin{array}{l}\text { La eliminación de una conducta anuladora causa o permite que continúe } \\
\text { un evento negativo. }\end{array}$ \\
\hline
\end{tabular}

Fuente: Elaboración propia.

CUADRO 7. TIPOS DE P S SEGÚN LA FUNCIÓN DE TRANSDUCCIÓN $\psi$

Vivencia de lo inefable $(\Omega)$

\begin{tabular}{cc}
\hline Mediación & Atestiguación \\
\hline$P_{23}$ & $P_{24}$ \\
\hline
\end{tabular}

Fuente: Elaboración propia.

\section{CUADRO 8. DESCRIPCIÓN DE LOS TIPOS DE P}

\begin{tabular}{lll} 
Tipos & $\begin{array}{c}\text { Relación funcional } \\
\operatorname{Exp}=\psi(\Omega)\end{array}$ & \multicolumn{1}{c}{ Significado } \\
\hline $\mathrm{P}_{23}$ & $\operatorname{Exp}^{\mathrm{M}}=\psi(\Omega)$ & $\begin{array}{l}\text { Mediación: O es objeto de la experiencia P al formar parte de ésta como } \\
\text { medio para su manifestación, mediante su cuerpo y conducta, de modo } \\
\text { voluntario o no, consciente o no. }\end{array}$ \\
\hline $\mathrm{P}_{24}$ & $\operatorname{Exp}^{\top}=\psi(\Omega)$ & $\begin{array}{l}\text { Atestiguación: O es sujeto pasivo de la experiencia P al formar parte de ésta o } \\
\text { no, mediante su presencia, de modo voluntario o no, consciente o no. }\end{array}$ \\
\hline
\end{tabular}

Fuente: Elaboración propia.

\section{TERCERA CLASE DE RELACIONES: RELACIONES ESPECIALES DE CAUSALIDAD (P CLASE $\chi$ )}

La tercera relación funcional entre $\mathrm{C}, \mathrm{E}$ y $\mathrm{O}$ también es de tipo causal. En este caso, $\mathrm{O}$ asume que la ejecución o cesación de una conducta suya genera o anula uno o más de los elementos de E, o actúa sobre $\mathrm{O}$, pero además de la ineficacia causal real, la atribución errónea de causalidad persiste a pesar de que se sabe que es errada. Los tipos posibles para $\mathrm{P}_{\chi}$ son formalmente equivalentes a los de $\mathrm{P}_{\alpha}$. En el cuadro 5, los que no son lógicamente 


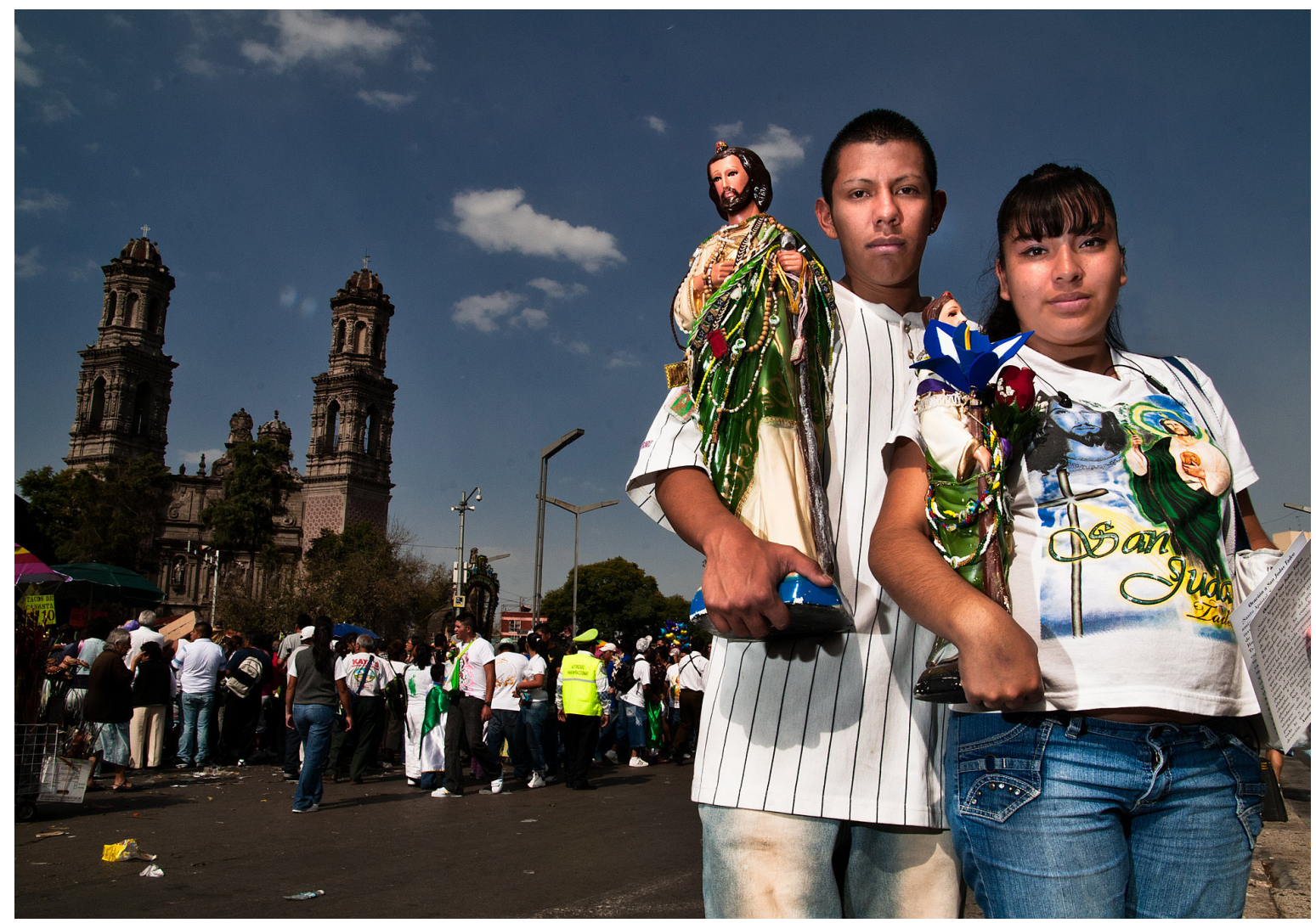

Rodrigo GonzÁlez • Devotos de San Judas Tadeo frente a la Iglesia de San Hipólito. Ciudad de México.

posibles se señalan con subíndices literales, en casillas sombreadas, y en el cuadro 6 se muestran los tipos resultantes.

\section{CUARTA CLASE DE RELACIONES: RELACIONES DE TRANSDUCCIÓN}

Ésta es la experiencia P: en este caso, lo que O experimenta como $\mathrm{P}$ puede ser comprensible o no para los otros, pero en todo caso amerita explicación, ya sea para su comprensión — parcial o completa - por parte de quien lo vivencia, ya para la comunicación de la experiencia vivida. En estos casos, el esfuerzo del aparato cognitivo de O se manifiesta en la transducción - la conversión de datos o información- de $\Omega$ - aquello que prima facie sería inexplicable pues contradice el funcionamiento ordinario del mundo: animales que hablan, estatuas que sangran, etc.- en lo explicable.

Al presentarse una relación funcional según la cual la experiencia de $\mathrm{P}$ sucede en función de la ocurrencia de lo inexplicable $[\operatorname{Exp}=\psi(\Omega)]$, se producen dos posibilidades básicas: una en la cual $\mathrm{O}$ es objeto de la experiencia $\mathrm{P}$, como medio para la manifestación de $\Omega$, esto es, como instrumento; y otra en la cual $\mathrm{O}$ es sujeto de la experiencia $\mathrm{P}$ como testigo u observador.

Así, operan dos clases de relaciones entre la experiencia y lo inefable, que dan lugar a dos clases de vivencia de $\mathrm{P}$ : la mediación y la atestiguación. Véanse los cuadros 7 y 8 . 


\section{RELACIONES MIXTAS O COMPUESTAS}

Las manifestaciones de P no necesariamente se presentan en tipos simples, pueden ocurrir casos concretos que impliquen dos o más tipos. Se presentan tres posibilidades: a) multiplicidad de tipos en progresión lineal, en la que la sucesión de conductas no incluye la repetición de conductas simples - por ejemplo, interpretación de un augurio-; b) multiplicidad de tipos en progresión no lineal, en la que la sucesión de conductas incluye la repetición de ciertas conductas simples hasta satisfacer una condición dada, lo que puede dar lugar a bucles — por ejemplo, en diversos rituales-, y c) composición de funciones, es decir, empleo del producto de una como objeto de otra - por ejemplo, interpretar un signo y luego manipular ese signo para generar un cambio-

\section{TIPOS DEFINITIVOS}

En el cuadro 9 se muestran los tipos que resultan de manera definitiva.

\section{UNA TIPOLOGÍA FENOMENOLÓGICA DE P}

Hasta aquí se ha mostrado el surgimiento de los tipos de $\mathrm{P}$ según las relaciones entre sus elementos, pero para llegar a sus distintas manifestaciones es preciso detectar unas formas que sirvan como enlace entre éstas y aquéllos. La solución que se propone es una tipología fenomenológica, referida a los fenómenos involucrados y no puramente a la representación simbólica.

Para ello se toman tres criterios de clasificación: a) tipo de ámbito; es decir, lo superior — asociado a lo luminoso, lo bueno y lo sagrado- y lo inferior — asociado a la oscuridad, la maldad y lo negativo-; b) entorno al cual se refieren las operaciones del sujeto; es decir, lo visible — mundo natural- y lo invisible
CUADRO 9. CLASES Y TIPOS DEFINITIVOS DE P

Clases y tipos de $\mathbf{P}$

Funciones

1) Clase $P_{a}$

\begin{tabular}{cl}
\hline$P_{1}$ & $E^{+}=\kappa(\mathrm{Cg})$ \\
\hline$P_{2}$ & $E^{-}=\kappa(\mathrm{Cg})$ \\
\hline$P_{3}$ & $\overline{E^{+}}=\kappa(\bar{C} g)$ \\
\hline$P_{4}$ & $\bar{E}^{-}=\kappa(\bar{C} g)$ \\
\hline$P_{5}$ & $\bar{E}^{+}=\kappa(\mathrm{Ca})$ \\
\hline$P_{6}$ & $\bar{E}^{-}=\kappa(\mathrm{Ca})$ \\
\hline$P_{7}$ & $E^{+}=\kappa(\bar{C} a)$ \\
\hline$P_{8}$ & $E^{-}=\kappa(\bar{C} a)$
\end{tabular}

2) Clase $P$

\begin{tabular}{cl}
\hline$P_{9}$ & $E_{p s}^{+}=\rho\left(E^{s}\right)$ \\
\hline$P_{10}$ & $E_{p r}^{+}=\rho\left(E^{s}\right)$ \\
\hline$P_{11}$ & $E_{f t}^{+}=\rho\left(E^{s}\right)$ \\
\hline$P_{12}$ & $E_{p s}^{-}=\rho\left(E^{s}\right)$ \\
\hline$P_{13}$ & $E_{p r}^{-}=\rho\left(E^{s}\right)$ \\
\hline$P_{14}$ & $E_{f t}^{-}=\rho\left(E^{s}\right)$ \\
\hline
\end{tabular}

3) Clase $P_{x}$

\begin{tabular}{|c|c|}
\hline$P_{15}$ & $\mathrm{E}^{+}=\varpi(\mathrm{Cg})$ \\
\hline$P_{16}$ & $\mathrm{E}^{-}=\varpi(\mathrm{Cg})$ \\
\hline$P_{17}$ & $\overline{\mathrm{E}}^{+}=\varpi(\overline{\mathrm{C}} \mathrm{g})$ \\
\hline $\mathrm{P}_{18}$ & $\overline{\mathrm{E}^{-}}=\varpi(\overline{\mathrm{C}} \mathrm{g})$ \\
\hline$P_{19}$ & $\overline{\mathrm{E}}^{+}=\varpi(\mathrm{Ca})$ \\
\hline$P_{20}$ & $\overline{\mathrm{E}^{-}}=\varpi(\mathrm{Ca})$ \\
\hline$P_{21}$ & $\mathrm{E}^{+}=\varpi(\overline{\mathrm{C}} \mathrm{a})$ \\
\hline$P_{22}$ & $\mathrm{E}^{-}=\varpi(\overline{\mathrm{C}} \mathrm{a})$ \\
\hline \multicolumn{2}{|c|}{ 4) Clase $P_{\delta}$} \\
\hline$P_{23}$ & $\operatorname{Exp}^{M}=\psi(\Omega)$ \\
\hline $\mathrm{P}_{24}$ & $\operatorname{Exp}^{\top}=\psi(\Omega)$ \\
\hline
\end{tabular}

Fuente: Elaboración propia.

-mundo sobrenatural- y c) tipo de conducta ejecutada, que puede ser de interpretación; de operación para la producción de efectos sobrenaturales; vivencial — de experiencia personal como medio o de presencia como testigo-. De lo que surgen 12 


\begin{tabular}{|c|c|c|c|c|}
\hline \multirow{2}{*}{ Ámbito* } & & \multicolumn{3}{|c|}{ Clase de actividad } \\
\hline & & Interpretativa & Operativa & Vivencial \\
\hline \multirow{2}{*}{$\begin{array}{l}\text { Ámbito } \\
\text { superior }\end{array}$} & $\begin{array}{l}\text { Entorno } \\
\text { visible }\end{array}$ & $\begin{array}{l}\text { Interpretación del entorno } \\
\text { visible superior (IVS) }\end{array}$ & $\begin{array}{l}\text { Operación sobre el entorno } \\
\text { visible superior (ovs) }\end{array}$ & $\begin{array}{l}\text { Experiencia paranormal en el } \\
\text { entorno visible superior (EVS) }\end{array}$ \\
\hline & $\begin{array}{l}\text { Entorno } \\
\text { invisible }\end{array}$ & $\begin{array}{l}\text { Interpretación del entorno } \\
\text { invisible superior (IIS) }\end{array}$ & $\begin{array}{l}\text { Operación sobre el entorno } \\
\text { invisible superior (oIs) }\end{array}$ & $\begin{array}{l}\text { Experiencia paranormal en el } \\
\text { entorno invisible superior (EIS) }\end{array}$ \\
\hline \multirow{2}{*}{$\begin{array}{l}\text { Ámbito } \\
\text { inferior }\end{array}$} & $\begin{array}{l}\text { Entorno } \\
\text { visible }\end{array}$ & $\begin{array}{l}\text { Interpretación del entorno } \\
\text { visible inferior (IVI) }\end{array}$ & $\begin{array}{l}\text { Operación sobre el entorno } \\
\text { visible inferior (ovI) }\end{array}$ & $\begin{array}{l}\text { Experiencia paranormal en el } \\
\text { entorno visible inferior (EVI) }\end{array}$ \\
\hline & $\begin{array}{l}\text { Entorno } \\
\text { invisible }\end{array}$ & $\begin{array}{l}\text { Interpretación del entorno } \\
\text { invisible inferior (III) }\end{array}$ & $\begin{array}{l}\text { Operación sobre el entorno } \\
\text { invisible inferior (oII) }\end{array}$ & $\begin{array}{l}\text { Experiencia paranormal en el } \\
\text { entorno invisible inferior (EII) }\end{array}$ \\
\hline
\end{tabular}

*El ámbito debe calificarse como positivo o bueno, o bien como negativo o malo, por quien ejecutó la conducta, interpretó el hecho o vivió la experiencia, no por el observador, testigo o analista.

Nota: No se incluye un ámbito neutral para no multiplicar los elementos de manera innecesaria.

Fuente: Elaboración propia.

formas de P. Lo que sucede posteriormente consiste en las diversas manifestaciones concretas de $\mathrm{P}$ (véase el cuadro 10).

\section{Niveles del modelo}

El modelo se compone de cuatro niveles, desde lo más abstracto hasta la manifestación empírica del fenómeno. El núcleo de $\mathrm{P}$ se compone de tres elementos - O, C y E- y las relaciones funcionales que existen entre ellos generan cuatro clases básicas de $\mathrm{P}$ - ésta es su estructura profunda- En en el primer nivel del modelo están las clases $\mathrm{P}_{\alpha}, \mathrm{P}_{\beta}, \mathrm{P}_{\chi}$ y $\mathrm{P}_{\delta}$, las cuales se manifiestan en 24 tipos - la primera estructura intermedia de $\mathrm{P}-\mathrm{y}$ constituyen el segundo nivel del modelo: los tipos de $\mathrm{P}\left(\mathrm{P}_{1} \ldots \mathrm{P}_{24}\right)$. $\mathrm{P}$ se presenta luego en ocho formas - la segunda estructura intermedia de $\mathrm{P}$-, que componen el tercer nivel del modelo: las formas de P (IVS ... EII).

La manifestación final de $\mathrm{P}$ presenta una amplia variedad de casos, que se aprecian en la actividad de los sujetos, ${ }^{9}$ según los distintos sistemas de creencias, características personales y culturas. Estos casos son la expresión observable o estructura superficial de $\mathrm{P}$ y constituyen el cuarto nivel del modelo: las prácticas de $\mathrm{P} .{ }^{10}$

Se propone que las estructuras deben presentar una relación de equivalencia $(\leftrightarrow)$ y deberían ser traducibles de un nivel al inmediato superior, puesto que cada una es resultado de la transformación de la anterior, por lo que debe conservarse una relación homeomórfica entre los conjuntos (véase la figura 1).

\section{Una definición de P}

Se define $\mathrm{P}$ como una estructura simbólica compuesta por $\mathrm{O}$-agentes humanos-, $\mathrm{C}$ - conjunto de conductas - y E - conjunto de agentes visibles Para una clasificación detallada de las supersticiones, véase Dundes (1961).

10 Aunque resultaría interesante, aquí no se pretende efectuar una analogía con la gramática generativa de Noam Chomsky (1965; 1995; 2002), debido a la naturaleza distinta de la gramática y la antropología, y porque el modelo chomskiano actual se ha modificado sensiblemente y ya no se postula tal distinción entre estructuras. 
FIGURA 1. SÍNTESIS GRÁFICA DEL MODELO DE LA PRÁCTICA DE LO PARANORMAL

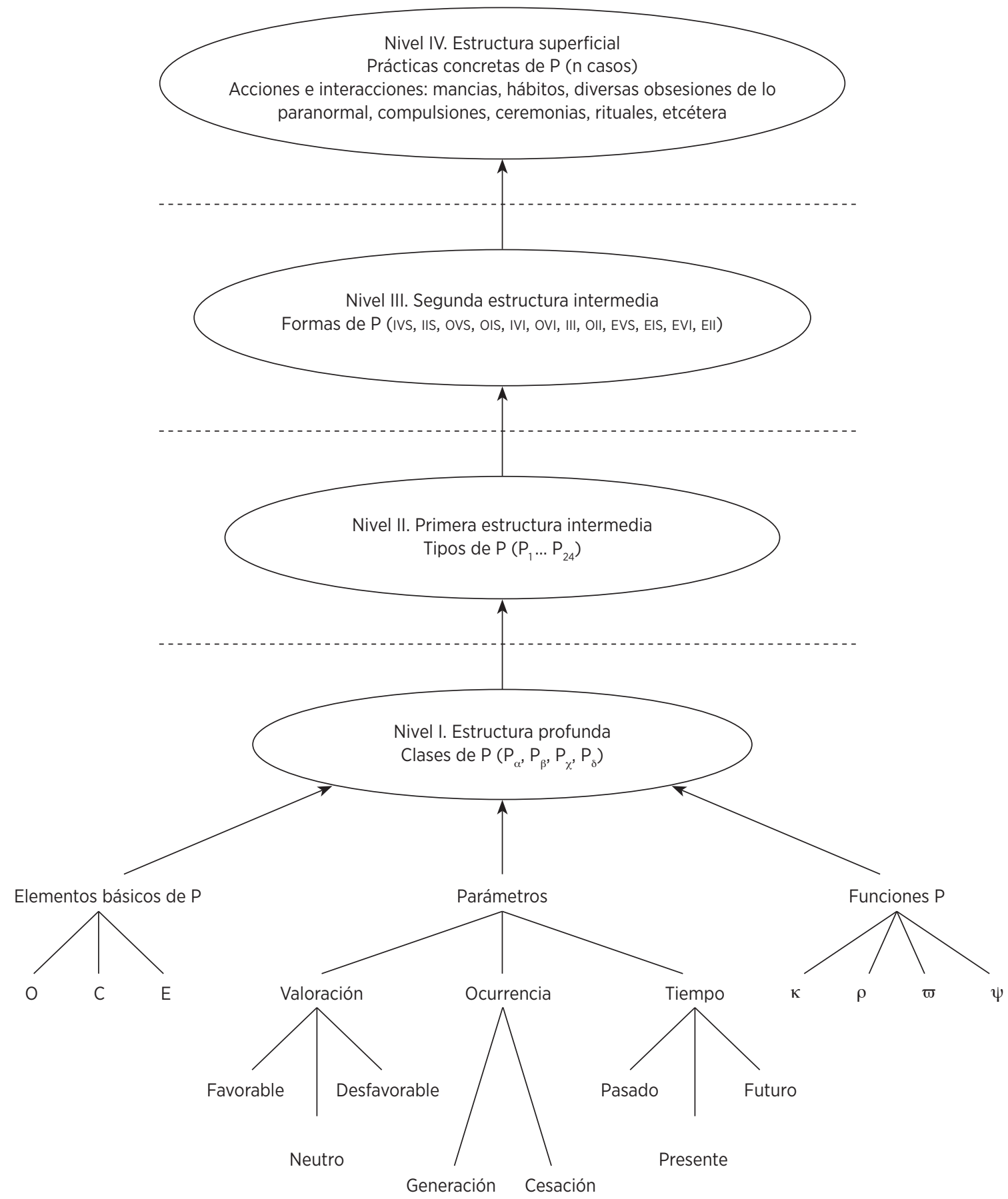

Fuente: Elaboración propia. 
e invisibles (I), hechos y eventos-, en la que $\mathrm{O}$ o I ejecutan u omiten uno o más componentes de C, en el contexto de lo inexplicable $(\Omega)$. Las relaciones entre $\mathrm{O}, \mathrm{E}$ y $\mathrm{C}$ implican relaciones lógicamente inadecuadas, aunque válidas para los $\mathrm{O}$ en el contexto $\Omega$.

C y E se encuentran restringidos por tres parámetros: tiempo — pasado, presente y futuro-; valor — favorable, neutro o desfavorable- y ocurrencia - generación o cesación-. Existen cuatro relaciones funcionales entre $\mathrm{O}, \mathrm{C}$ y $\mathrm{E}$ dentro de $\mathrm{E}$, inadecuadas desde un punto de vista lógico: 1) relaciones causales $(\kappa)$, que pueden ser generativas o anuladoras y sólo pueden darse desde $\mathrm{C}$ hacia $\mathrm{E}$ y $\mathrm{O} ; 2$ ) relaciones correlacionales ( $\rho$ ), que sólo pueden darse en E; 3 ) relaciones causales, generativas o anuladoras (๘), desde $\mathrm{C}$ hacia $\mathrm{E}$ y O, percibidas como ilógicas por $\mathrm{O}$, y 4) relaciones transductoras $(\psi)$, que se dan entre $\mathrm{O}$ y $\Omega$, en las cuales la experiencia de $\mathrm{O}$ surge como resultado transducido de $\Omega$.

\section{Conclusión}

En esta investigación se han analizado las clases y tipos de práctica de lo paranormal $(\mathrm{P})$ con fundamento en las relaciones funcionales que se presentan en las operaciones cognitivas que ejecuta un organismo y que pueden ser representadas matemáticamente. Dicha fundamentación no había sido propuesta anteriormente. Así, se ha adelantado una solución al problema de crear una tipología de P no meramente intuitiva ni casuística.

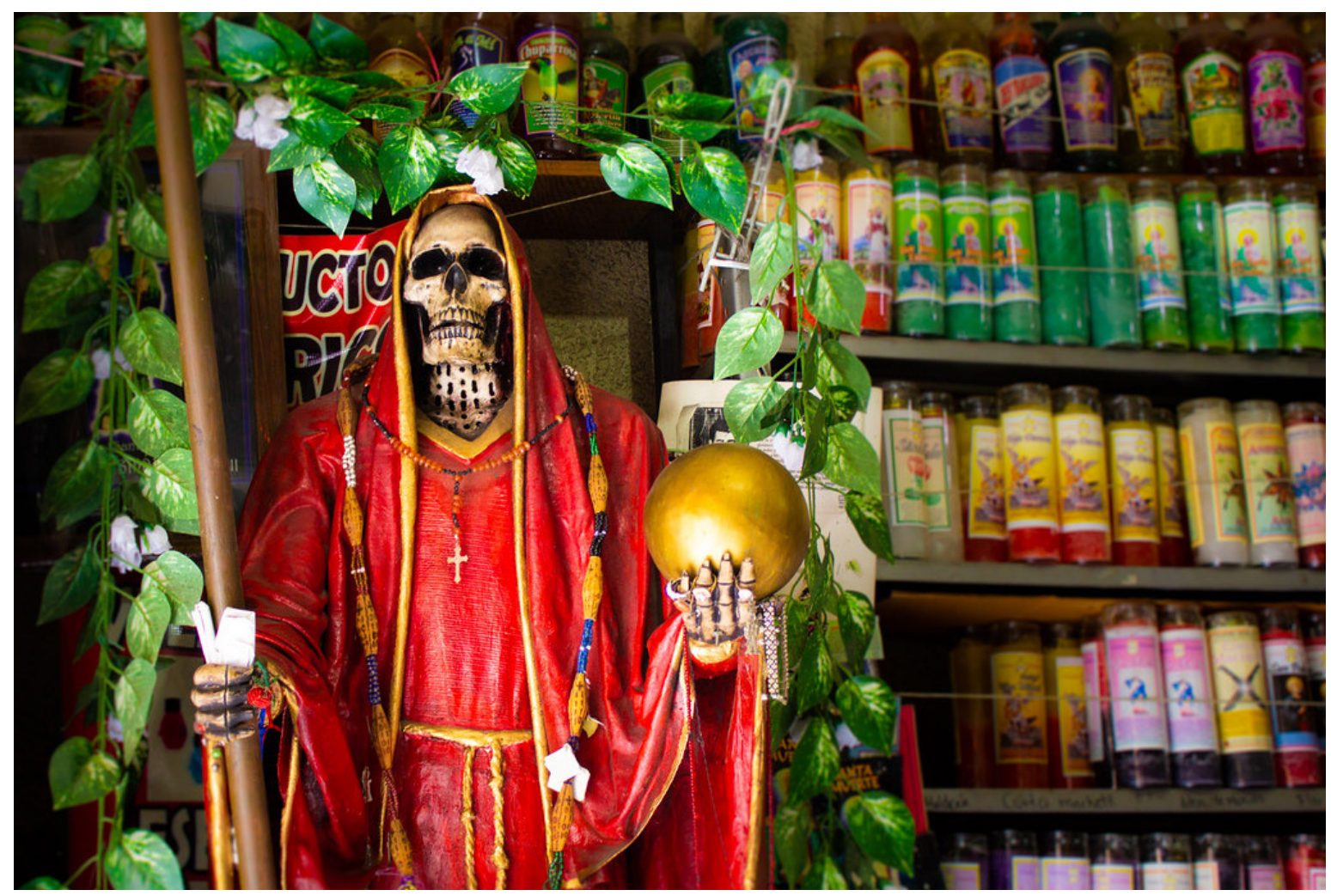

CAT CALHOUn/FLICKR • Santa Muerte. 
La existencia real de las estructuras encontradas es algo que no puede ser dilucidado de manera teórica, sino empírica; de modo que, aunque aquí se propone un modelo, éste deberá ser sometido a prueba posteriormente. Así, este modelo no pretende que se prescinda del análisis emic, sino mostrar que aun cuando las $\mathrm{P}$ se manifiestan en diversas culturas, a todas subyace una estructura que en sus formas locales deberá presentar sólo variaciones superficiales, es decir, de manifestación empírica y dependientes de la cultura.

Este modelo abre vías de sumo interés para la investigación, mediante la observación de $\mathrm{P}$, entrevistas, análisis de textos, etc. Dichas vías son: 1) análisis de la existencia de las estructuras propuestas; 2) determinación de si puede darse, y bajo qué condiciones, una composición matemática de las funciones cuando se presenta un tipo mixto de $\mathrm{P}$; 3 ) examen de la probabilidad de predecir una clase, tipo o forma de $\mathrm{P}$, dada la ejecución de otra, en series de conductas rituales y no rituales; 4) verificación o refutación en campo de la estructura de $\mathrm{P}$ propuesta; 5) análisis de la validez del modelo para épocas diferentes, en textos históricos, y 6) predominio cultural de tipos de $\mathrm{P}$, según relaciones de asociación o correlación entre la tipología y las características culturales. D

\section{Bibliografía}

Beck, Jan y Wolfgang Forstmeier, 2007, "Superstition and Belief as Inevitable By-Products of an Adaptive Learning Strategy", en Human Nature, vol. 18, núm. 1, pp. 35-46. Dol: <https://doi.org/10.1007/bf02820845>.

Brugger, Peter y Isabelle Viaud-Delmont, 2010, "Superstitiousness in Obsessive-Compulsive Disorder", en Dialogues in Clinical Neuroscience, vol. 12, núm. 2, pp. 250-254. Disponible en línea: <http://www.ncbi.nlm.nih.gov/pmc/articles/PMC3181957/pdf/DialoguesClinNeurosci-12250.pdf>.

Bruno, Giordano, 2007 [1588; 1590], De la magia. De los vínculos en general, Cactus, Buenos Aires.

Campbell, Colin, 1996, "Half-Belief and the Paradox of Ritual Instrumental Activism: A Theory of Modern Superstition", en The British Journal of Sociology, vol. 47, núm. 1, pp. 151-166.

Chomsky, Noam, 1965, Aspects of the Theory of Syntax, Mit Press, Cambridge.

_ 1995, The Minimalist Program, MiT Press, Cambridge.

2002, On Nature and Language, Cambridge University Press, Cambridge.

D’Agati, Marina, 2014, “'I Feel Like I’m Going to Win': Superstition in Gambling”, en Qualitative Sociology Review, vol. 10, núm. 2, pp. 80-101. Disponible en línea: <http://www.qualitativesociologyreview.org/ENG/archive_eng.php>.

Dundes, Alan, 1961, "Brown County Superstitions”, en Midwest Folklore, núm. 11, pp. 25-56.

Foster, Kevin y Hanna Kokko, 2009, "The Evolution of Superstitious and Superstition-Like Behaviour", en Proceedings of the Royal Society, núm. B 276, pp. 31-37. Dol: <http://10.1098/rspb.2008.0981>.

Frater, U. D., 2005, High Magic: Theory and Practice, Llewellyn, Woodbury.

George, Sonia y Krishna Sreedhar, 2006, "Globalisation and the Prevalence of Superstitious Beliefs", en Journal of the Indian Academy of Applied Psychology, vol. 32, núm. 3, pp. 241-247. Disponible en línea: <http://medind.nic.in/jak/t06/i1/jakt06ilp241.pdf>.

Greenwood, Susan, 2000, Magic, Witchcraft and the Otherworld, Berg, Oxford.

Jarvis, Peter, 1980, “Towards a Sociological Understanding of Superstition”, en Social Compass, vol. 27, núm. 2-3, pp. 285-295.

Kramer, Heinrich y Jacobus Sprenger, 1996 [1486], Malleus maleficarum, Bracken, Londres.

Leuba, James, 1912, "The Varieties, Classification, and Origin of Magic”, en American Anthropologist, vol. 14, núm. 2, pp. 350-367. Disponible en línea: <http://www.jstor.org/stable/659937>.

Lindeman, Marjaana y Kia Aarnio, 2007, "Superstitious, Magical, and Paranormal Beliefs: An Integrative Model”, en Journal of Research in Personality, vol. 41, núm. 4, pp. 731-744. Dol: <https://10.1016/j.jrp.2006.06.009>.

MacIntyre, Alasdair, 1981, After Virtue. A Study in Moral Theory, University of Notre Dame Press, Notre Dame. 
Markle, Thomas, 2010, "The Magic that Binds Us: Magical Thinking and Inclusive Fitness", en Journal of Social, Evolutionary, and Cultural Psychology, vol. 4, núm. 1, pp. 18-33. Dol: <https://doi.org/10.1037/h0099304>.

Matute, Helena y Miguel Vadillo, 2012, "Causal Learning and Illusions of Control”, en Norbert Seel (ed.), Encyclopedia of the Sciences of Learning, Springer, Berlin, pp. 1-4. Disponible en línea: <https://10.1007/SpringerReference_301904>.

Melchior-Bonnet, Sabine, 2001, The Mirror. A History, Routledge, Nueva York.

Murray, Margaret, 2006, El dios de los brujos, Fondo de Cultura Económica, México.

Näyhä, Simo, 2002, "Traffic Deaths and Superstition on Friday the 13"th”, en American Journal of Psychiatry, vol. 159, núm. 12, pp. 2110-2111. Dol: <https://doi.org/10.1176/appi.ajp.159.12.2110>.

Oberter, Rachel, 2012, “'The Sublimation of Matter into Spirit': Anna Mary Howitt's Automatic Drawings”, en Sarah Willburn y Tatiana Kontou (eds.), The Ashgate Research Companion to Nineteenth-Century Spiritualism and The Occult, Ashgate Publishing Limited, Surrey, pp. 333-358.

Otto, Bernd-Christian y Michael Stausberg, 2014, "General Introduction”, en Bernd-Christian Otto y Michael Stausberg (eds.), Defining Magic: A Reader, Routledge, Nueva York, pp. 1-13.

Pronin, Emily, Daniel Wegner, Kimberly McCarthy y Sylvia Rodríguez, 2006, "Everyday Magical Powers: The Role of Apparent Mental Causation in the Overestimation of Personal Influence", en Journal of Personality and Social Psychology, vol. 91, núm. 2, pp. 218-231. Dol: <https://doi.org/10.1037/0022-3514.91.2.218>.

Sándor, László, 2007, Anglo-Saxon Prognostics, 900-1100. Study and Texts, Brill, Leiden.

Simonds, Laura, James Demetre y Cristina Read, 2009, "Relationships between Magical Thinking, Obsessive-Compulsiveness and Other Forms of Anxiety in a Sample of Non-Clinical Children”, en British Journal of Developmental Psychology, vol. 27, núm. 2, pp. 457-71. Dol: <https://doi.org/10.1348/026151008×345582>

Skinner, Burrhus, 1948, “'Superstition' in the Pigeon”, en Journal of Experimental Psychology, núm. 38, pp. 168-172. Dol: <https://doi.org/10.1037/ h0055873>.

Spears, Lauren, 2013, An Examination of Magical Beliefs as Predictors of Obsessive-Compulsive Symptom Dimensions, tesis de doctorado en psicología, University of Kansas. Disponible en línea: <https://kuscholarworks.ku.edu/bitstream/handle/1808/16830/Spears_ ku_0099D_12740_DATA_1.pdf?sequence=1>.

Stevens, Phillips, 2004, “Divination”, en Frank Salamone (ed.), Encyclopedia of Religious Rites, Rituals, and Festivals, Routledge, Londres, pp. 114-118.

Vadillo, Miguel, Helena Matute y Fernando Blanco, 2013, "Fighting the Illusion of Control: How to Make Use of Cue Competition and Alternative Explanations", en Universitas Psychologica, vol. 12, núm. 1, pp. 261-270. 